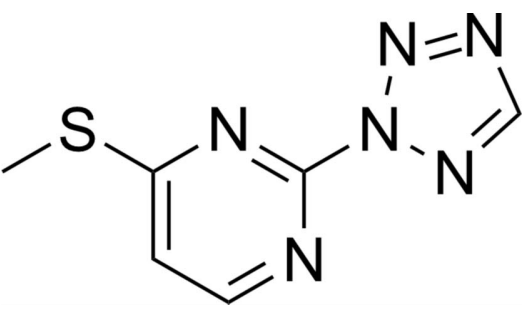

\title{
Crystal structure of 4-methylsulfanyl-2- (2H-tetrazol-2-yl)pyrimidine
}

\section{Andreas Thomann, ${ }^{\mathrm{a}}$ Volker Huch ${ }^{\mathrm{b}}$ and Rolf W. Hartmann $^{\mathrm{a}, \mathrm{c} *}$}

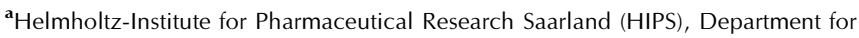
Drug Design and Optimization (DDOP), Saarland University, Campus E8.1, D-66123 Saarbruecken, Germany, ${ }^{\mathbf{b}}$ Department of Inorganic Chemistry, Saarland University, Campus B2.2, D-66123 Saarbruecken, Germany, and ${ }^{\mathrm{c}}$ Department of Pharmacy, Pharmaceutical and Medicinal Chemistry, Saarland University, Campus C2.3, D-66123 Saarbruecken, Germany. *Correspondence e-mail:

rolf.hartmann@helmholtz-hzi.de

Received 1 December 2015; accepted 9 December 2015

Edited by H. Stoeckli-Evans, University of Neuchâtel, Switzerland

The title compound, $\mathrm{C}_{6} \mathrm{H}_{6} \mathrm{~N}_{6} \mathrm{~S}$, crystallized with two independent molecules $(A$ and $B)$ in the asymmetric unit. The conformation of the two molecules differs slightly. While the tetrazole ring is inclined to the pyrimidene ring by 5.48 (7) and $4.24(7)^{\circ}$ in molecules $A$ and $B$, respectively, the $\mathrm{N}-\mathrm{C}-\mathrm{S}-\mathrm{C}$ torsion angles of the thiomethyl groups differ by $c a 180^{\circ}$. In the crystal, the $A$ and $B$ molecules are linked via a $\mathrm{C}-\mathrm{H} \cdots \mathrm{N}$ hydrogen bond. They stack along the $b$-axis direction forming columns within which there are weak $\pi-\pi$ interactions present [shortest inter-centroid distance $=3.6933(13) \AA$ ]

Keywords: crystal structure; tetrazole; pyrimidine; thio; heterocyles; $\mathrm{S}_{\mathrm{N}} \mathrm{Ar}$ reactions; $\pi-\pi$ interactions.

CCDC reference: 1441424

\section{Related literature}

For applications of tetrazolyl-substituted aromatic systems in metal-ligand research, see: Kim et al. (2008); Stoessel et al. (2010); in drug development, see: Pasternak et al. (2012); Biswas et al. (2015); in polymer synthesis, see: Yu et al. (2008); Sengupta et al. (2010). For the synthesis of 4-methylsulfanyl-2(1H-tetrazol-1-yl)pyrimidine and the title compound, see: Thomann et al. (2014).

\section{Experimental}

\subsection{Crystal data}

$\mathrm{C}_{6} \mathrm{H}_{6} \mathrm{~N}_{6} \mathrm{~S}$

$M_{r}=194.23$

Triclinic, $P \overline{1}$

$a=6.3001(17) \AA$

$b=7.393$ (2) A

$c=18.159(5) \AA$

$\alpha=91.407(7)^{\circ}$

$\beta=95.864(7)^{\circ}$

\subsection{Data collection}

Bruker APEXII CCD

diffractometer

Absorption correction: multi-scan (SADABS; Bruker, 2010)

$T_{\min }=0.716, T_{\max }=0.746$

\subsection{Refinement}

$R\left[F^{2}>2 \sigma\left(F^{2}\right)\right]=0.034$

$w R\left(F^{2}\right)=0.086$

$S=1.01$

4581 reflections

$$
\begin{aligned}
& \gamma=102.695(8)^{\circ} \\
& V=819.9(4) \AA^{3} \\
& Z=4 \\
& \text { Mo } K \alpha \text { radiation } \\
& \mu=0.35 \mathrm{~mm}^{-1} \\
& T=143 \mathrm{~K} \\
& 0.22 \times 0.22 \times 0.01 \mathrm{~mm}
\end{aligned}
$$

15501 measured reflections 4581 independent reflections 3596 reflections with $I>2 \sigma(I)$ $R_{\text {int }}=0.028$

Table 1

Hydrogen-bond geometry $\left(\AA{ }^{\circ}\right)$.

\begin{tabular}{lllll}
\hline$D-\mathrm{H} \cdots A$ & $D-\mathrm{H}$ & $\mathrm{H} \cdots A$ & $D \cdots A$ & $D-\mathrm{H} \cdots A$ \\
\hline $\mathrm{C} 2-\mathrm{H} 1 \cdots \mathrm{N} 9^{\mathrm{i}}$ & $0.89(2)$ & $2.58(2)$ & $3.203(2)$ & $129(2)$ \\
\hline
\end{tabular}

Symmetry code: (i) $x-1, y, z$.

Data collection: APEX2 (Bruker, 2010); cell refinement: SAINT (Bruker, 2010); data reduction: $S A I N T$; $\operatorname{program}(\mathrm{s})$ used to solve structure: SHELXS97 (Sheldrick 2008); program(s) used to refine structure: SHELXL2014 (Sheldrick, 2015); molecular graphics: PLATON (Spek, 2009); software used to prepare material for publication: SHELXL2014 and PLATON.

\section{Acknowledgements}

We thank Nadja Klippel for the synthesis of the title compound.

Supporting information for this paper is available from the IUCr electronic archives (Reference: SU5253). 


\section{References}

Biswas, D., Ding, F.-X., Dong, S., Gu, X., Jiang, J., Pasternak, A., Suzuki, T., Vacca, J. \& Xu, S. (2015). PCT Int. Appl. WO2015103756 A1.

Bruker (2010). APEX2, SAINT and SADABS. Bruker AXS Inc., Madison, Wisconsin, USA.

Kim, Y.-J., Lee, K.-E., Jeon, H.-T., Huh, H. S. \& Lee, S. W. (2008). Inorg. Chim. Acta, 361, 2159-2165.

Pasternak, A., Dejesus, R. K., Zhu, Y., Yang, L., Walsh, S., Pio, B., Shahripour, A., Tang, H., Belyk, K. \& Kim, D. (2012). PCT Int. Appl. WO 2012058134 A1.
Sengupta, O. \& Mukherjee, P. S. (2010). Inorg. Chem. 49, 8583-8590.

Sheldrick, G. M. (2008). Acta Cryst. A64, 112-122.

Sheldrick, G. M. (2015). Acta Cryst. C71, 3-8.

Spek, A. L. (2009). Acta Cryst. D65, 148-155.

Stoessel, P., Heil, H., Jooseten, D., Pflumm, C., Gerhard, A. \& Breuning, E. (2010). PCT Int. Appl. WO 2010086089 A1.

Thomann, A., Börger, C., Empting, M. \& Hartmann, R. W. (2014). Synlett, 25, 935-938.

Yu, L., Zhang, Z., Chen, X., Zhang, W., Wu, J., Cheng, Z., Zhu, J. \& Zhu, X. (2008). J. Polym. Sci. A Polym. Chem. 46, 682-691. 


\section{supporting information}

Acta Cryst. (2015). E71, o1051-o1052 [https://doi.org/10.1107/S2056989015023634]

\section{Crystal structure of 4-methylsulfanyl-2-(2H-tetrazol-2-yl)pyrimidine}

\section{Andreas Thomann, Volker Huch and Rolf W. Hartmann}

\section{S1. Comment}

4-tetrazolylpyrimidines are well reported scaffolds in many bioactive entities. Besides synthetic chemistry, tetrazolyl substituted aromatic systems are also of high interest for example, in metal-ligand research (Kim et al., 2008; Stoessel et al., 2010), drug development (Pasternak et al., 2012; Biswas et al., 2015) and polymer discovery (Yu et al., 2008; Sengupta et al., 2010). Thus, the knowledge of the three dimensional structure of these moieties is of crucial importance for the rational design in these fields of research. Recently, we have reported a novel method to synthesize such compounds (Thomann et al., 2014). We have reported the synthesis of 4-(methylthio)-2-(1H-tetrazol-1-yl)pyrimidine (1). Interestingly, when scaling up the reaction, another product was found in small amounts. NMR analytical characterization revealed the compound to be the 2-tetrazolyl regioisomer (2). To determine unequivocally proof of the structure of this compound, we determined its crystal structure.

The title compound (2), crystallized with two independent molecules (A and B) in the asymmetric unit (Fig. 1). Interestingly, the two molecules differ in their conformation. While the tetrazole moieties are arranged similarly, with the tetrazole ring is inclined to the pyrimidene ring by 5.48 (7) and 4.24 (7) ${ }^{\circ}$ in molecules A and B, respectively, the thiomethyl groups have a difference of the torsion angle about the $\mathrm{C}_{\mathrm{ar}} \cdots \mathrm{S}$ bond of ca. $180^{\circ}$ [for example, torsion angle $\mathrm{N} 5-$ $\mathrm{C} 4-\mathrm{S} 1-\mathrm{C} 6=0.89(12)^{\circ}$, compared to torsion angle $\left.\mathrm{N} 11-\mathrm{C} 10-\mathrm{S} 2-\mathrm{C} 12=-176.78(10)^{\circ}\right]$ indicating higher rotational freedom than the tetrazoles (Fig. 1). The latter finding is of importance for computational chemists in medicinal chemistry, as the polarized hydrogen at atom $\mathrm{C} 5$ of the tetrazole ring is able to form non-classical hydrogen bonds. Therefore, the results from the crystal structure may favour this conformational isomer for in silico predictions. In the crystal, the $\mathrm{A}$ and $\mathrm{B}$ molecules are linked via a $\mathrm{C}-\mathrm{H} \cdots \mathrm{N}$ hydrogen bond (Table 1 and Fig. 2). They stack along the $b$ axis direction forming columns within which there are weak $\pi-\pi$ interactions present [shortest inter-centroid distance is $\mathrm{Cg} 2 \cdots \mathrm{Cg} 4{ }^{\mathrm{i}}=3.6918$ (5) $\AA$; $\mathrm{Cg} 2$ and $\mathrm{Cg} 4$ are the centroids of rings $\mathrm{N} 5 / \mathrm{N} 6 / \mathrm{C} 1-\mathrm{C} 4$ and $\mathrm{N} 11 / \mathrm{N} 12 / \mathrm{C} 7-\mathrm{C} 10$, respectively; symmetry code: (i) $\mathrm{x}, \mathrm{y}+1, \mathrm{z}]$.

\section{S2. Synthesis and crystallization}

The title compound (2), was synthesized following a previously reported procedure (Thomann et al., 2014). A mixture of 4-chloro-2-(methylthio)pyrimidine, $1 H$-tetrazole and triethylamine, in the ratio 1:1:1, was stirred under microwave irradiation at $50 \mathrm{~W}, 353 \mathrm{~K}$ for $1 \mathrm{~h}$. The crude product was purified by flash chromatography (hexane:ethyl acetate, 8:2, $\mathrm{R}_{\mathrm{f}}$ $=0.25$ ) to yield a white solid (9\%). Crystals formed at $294 \mathrm{~K}$ after $16 \mathrm{~h}$ from a saturated solution of 2 in ethyl acetate. ${ }^{1} \mathrm{H}$ $\operatorname{NMR}\left(\mathrm{CDCl}_{3}, 300 \mathrm{MHz}\right) 8.80(\mathrm{dd}, J=5.3,0.6 \mathrm{~Hz}, 1 \mathrm{H}), 8.77(\mathrm{~s}, 1 \mathrm{H}), 7.77(\mathrm{dd}, J=5.3,0.7 \mathrm{~Hz}, 1 \mathrm{H}), 2.69 \mathrm{ppm}(\mathrm{d}, J=$ $0.7 \mathrm{~Hz}, 3 \mathrm{H})$. 


\section{S3. Refinement}

Crystal data, data collection and structure refinement details are summarized in Table 2. $\mathrm{H}$ atoms were located in a difference Fourier map and freely refined.

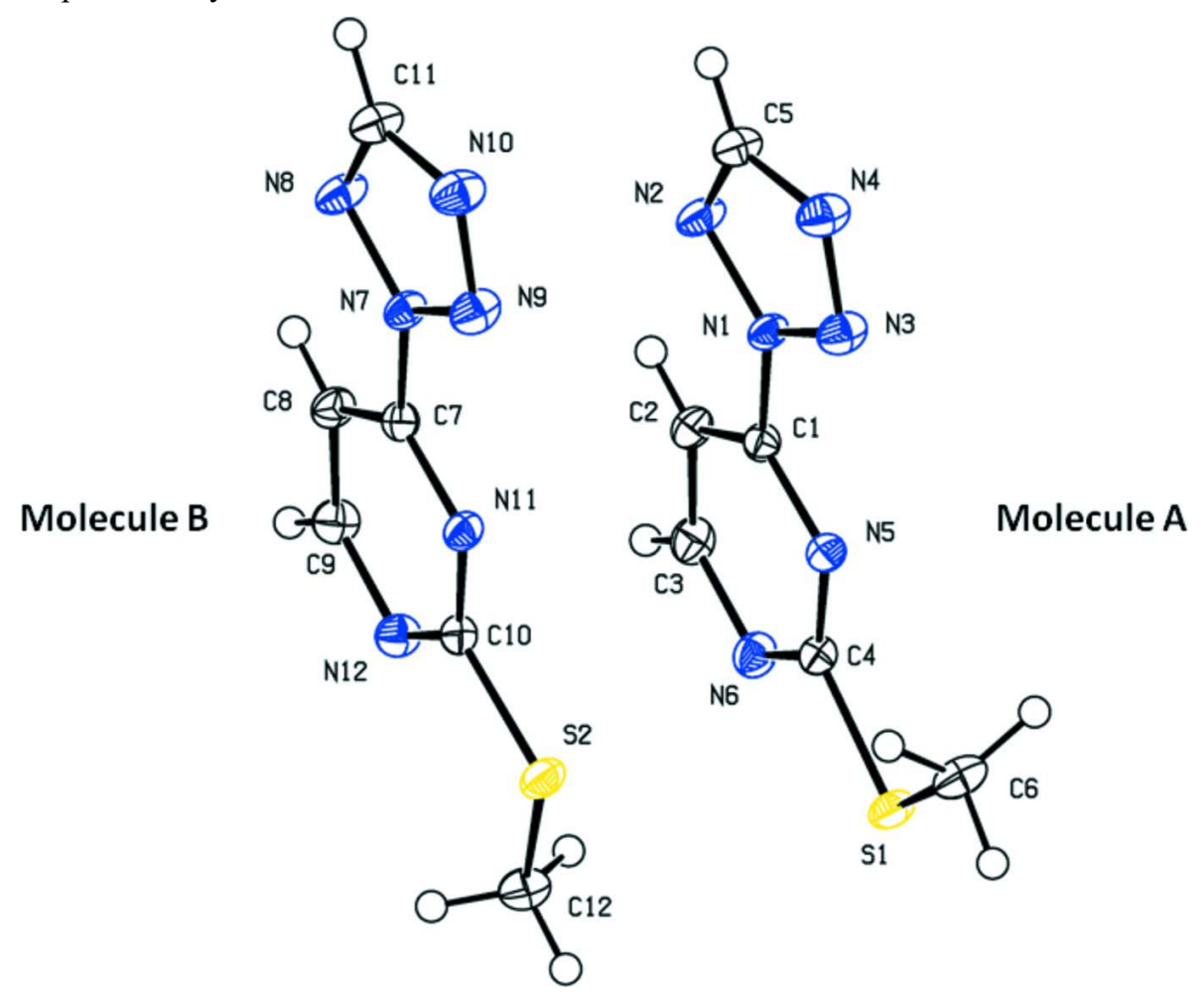

Figure 1

The molecular structure of the two independent molecules $(A$ and $B)$ of the title compound (2), with atom labelling. Displacement ellipsoids are drawn at the $50 \%$ probability level.

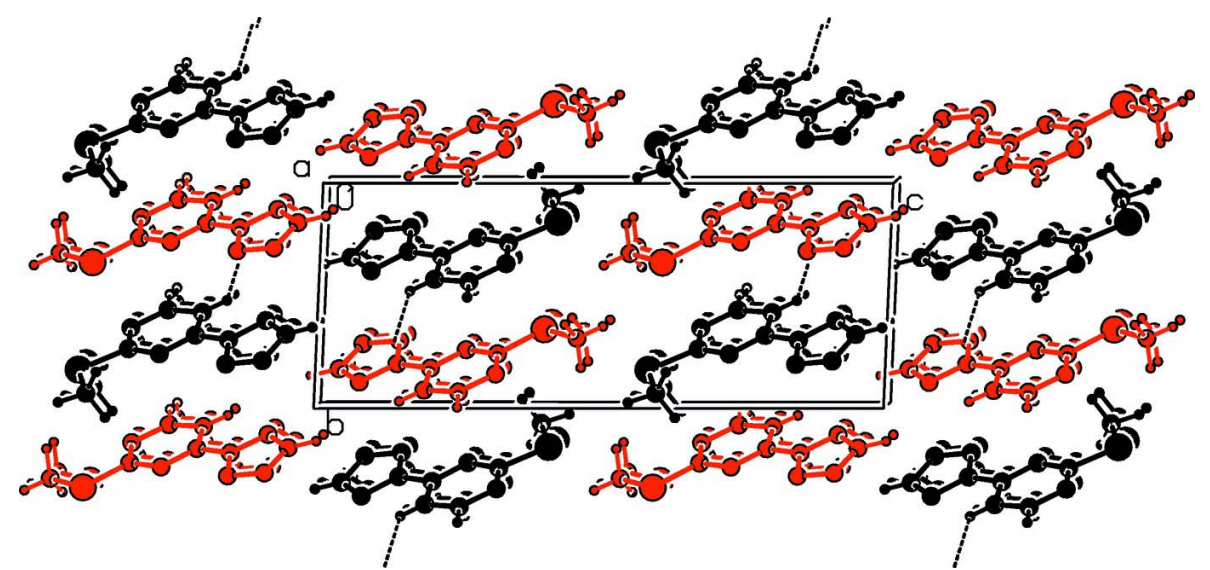

Figure 2

The crystal packing of the two independent molecules ( $A$ black; $B$ red) of the title compound (2), viewed along the $a$ axis. Hydrogen bonds are shown as dashed lines (see Table 1). 
<smiles>CSc1ccnc(-n2cnnn2)n1</smiles>

1

Figure 3

Compounds (1) and (2).

4-Methylsulfanyl-2-(2H-tetrazol-2-yl)pyrimidine

Crystal data

\section{$\mathrm{C}_{6} \mathrm{H}_{6} \mathrm{~N}_{6} \mathrm{~S}$}

$M_{r}=194.23$

Triclinic, $P \overline{1}$

$a=6.3001$ (17) $\AA$

$b=7.393(2) \AA$

$c=18.159(5) \AA$

$\alpha=91.407(7)^{\circ}$

$\beta=95.864(7)^{\circ}$

$\gamma=102.695(8)^{\circ}$

$V=819.9(4) \AA^{3}$

\section{Data collection}

Bruker APEXII CCD

diffractometer

$\varphi$ and $\omega$ scans

Absorption correction: multi-scan

(SADABS; Bruker, 2010)

$T_{\min }=0.716, T_{\max }=0.746$

15501 measured reflections

\section{Refinement}

Refinement on $F^{2}$

Least-squares matrix: full

$R\left[F^{2}>2 \sigma\left(F^{2}\right)\right]=0.034$

$w R\left(F^{2}\right)=0.086$

$S=1.01$

4581 reflections

283 parameters

0 restraints<smiles>CSc1ccnc(-n2ncnn2)n1</smiles>

2
$Z=4$

$F(000)=400$

$D_{\mathrm{x}}=1.574 \mathrm{Mg} \mathrm{m}^{-3}$

Mo $K \alpha$ radiation, $\lambda=0.71073 \AA$

Cell parameters from 728 reflections

$\theta=3.6-24.3^{\circ}$

$\mu=0.35 \mathrm{~mm}^{-1}$

$T=143 \mathrm{~K}$

Cuboid, colourless

$0.22 \times 0.22 \times 0.01 \mathrm{~mm}$

4581 independent reflections 3596 reflections with $I>2 \sigma(I)$

$R_{\text {int }}=0.028$

$\theta_{\text {max }}=29.6^{\circ}, \theta_{\min }=2.3^{\circ}$

$h=-8 \rightarrow 8$

$k=-10 \rightarrow 10$

$l=-24 \rightarrow 25$

Hydrogen site location: difference Fourier map

All $\mathrm{H}$-atom parameters refined

$$
\begin{aligned}
& w=1 /\left[\sigma^{2}\left(F_{\mathrm{o}}^{2}\right)+(0.0376 P)^{2}+0.2718 P\right] \\
& \text { where } P=\left(F_{\mathrm{o}}^{2}+2 F_{\mathrm{c}}^{2}\right) / 3 \\
& (\Delta / \sigma)_{\max }=0.001 \\
& \Delta \rho_{\max }=0.35 \mathrm{e} \AA^{-3} \\
& \Delta \rho_{\min }=-0.30 \mathrm{e} \AA^{-3}
\end{aligned}
$$

Special details

Geometry. All esds (except the esd in the dihedral angle between two 1.s. planes) are estimated using the full covariance matrix. The cell esds are taken into account individually in the estimation of esds in distances, angles and torsion angles; correlations between esds in cell parameters are only used when they are defined by crystal symmetry. An approximate (isotropic) treatment of cell esds is used for estimating esds involving l.s. planes. 
Fractional atomic coordinates and isotropic or equivalent isotropic displacement parameters $\left(\AA^{2}\right)$

\begin{tabular}{|c|c|c|c|c|}
\hline & $x$ & $y$ & $z$ & $U_{\text {iso }} * / U_{\text {eq }}$ \\
\hline $\mathrm{S} 1$ & $0.84117(6)$ & $0.83334(5)$ & $0.58281(2)$ & $0.01975(9)$ \\
\hline N1 & $1.04303(18)$ & $0.69484(15)$ & $0.84617(6)$ & $0.0152(2)$ \\
\hline N2 & 1.00906 (19) & $0.60999(17)$ & $0.90974(6)$ & $0.0208(3)$ \\
\hline N3 & 1.24517 (19) & $0.79683(17)$ & $0.84553(7)$ & 0.0209 \\
\hline N4 & $1.3498(2)$ & $0.77865(18)$ & $0.91059(7)$ & 0.0233 \\
\hline N5 & $0.93885(18)$ & $0.76012(15)$ & $0.72442(6)$ & $0.0159(2)$ \\
\hline N6 & $0.56784(19)$ & $0.65519(16)$ & $0.66803(7)$ & 0.0200 \\
\hline $\mathrm{C} 1$ & $0.8749(2)$ & $0.67900(17)$ & $0.78527(7)$ & 0.0148 \\
\hline $\mathrm{C} 2$ & $0.6647(2)$ & $0.58341(19)$ & $0.79335(8)$ & 0.0184 \\
\hline $\mathrm{C} 3$ & $0.5146(2)$ & $0.5770(2)$ & $0.73096(8)$ & 0.0203 \\
\hline $\mathrm{C} 4$ & $0.7801(2)$ & $0.74205(18)$ & $0.66783(8)$ & $0.0162(3)$ \\
\hline $\mathrm{C} 5$ & $1.2034(2)$ & $0.6651(2)$ & $0.94818(8)$ & 0.0209 \\
\hline C6 & $1.1309(2)$ & $0.9298(2)$ & $0.60068(9)$ & 0.0230 \\
\hline H1 & $0.629(3)$ & $0.532(2)$ & $0.8352(10)$ & $0.028(5)^{*}$ \\
\hline $\mathrm{H} 2$ & $0.366(3)$ & $0.512(2)$ & $0.7313(9)$ & $0.023(4)^{*}$ \\
\hline H3 & $1.235(3)$ & $0.634(2)$ & $0.9944(11)$ & $0.030(5)^{*}$ \\
\hline $\mathrm{H} 4$ & $1.204(3)$ & $0.833(2)$ & $0.6178(10)$ & $0.032(5)^{*}$ \\
\hline H5 & $1.160(3)$ & $1.037(2)$ & $0.6366(10)$ & $0.030(5)^{*}$ \\
\hline H6 & $1.172(3)$ & $0.969(3)$ & $0.5531(11)$ & $0.040(5)^{*}$ \\
\hline $\mathrm{S} 2$ & $0.91337(6)$ & $0.35407(5)$ & $0.59998(2)$ & $0.02003(10)$ \\
\hline N7 & $1.07557(18)$ & $0.19569(15)$ & $0.85809(6)$ & $0.0156(2)$ \\
\hline N8 & $1.0376(2)$ & 0.11547 (17) & $0.92240(7)$ & 0.0219 \\
\hline N9 & $1.28032(19)$ & $0.29223(17)$ & $0.85739(7)$ & 0.0218 \\
\hline N10 & $1.3817(2)$ & $0.27602(18)$ & $0.92304(7)$ & 0.0241 \\
\hline N11 & $0.97742(18)$ & $0.26119(15)$ & $0.73636(6)$ & $0.0159(2)$ \\
\hline N12 & 0.60819 (19) & $0.16413(16)$ & $0.67866(7)$ & $0.0192(2)$ \\
\hline $\mathrm{C} 7$ & $0.9101(2)$ & $0.18161(17)$ & $0.79690(7)$ & 0.0146 \\
\hline $\mathrm{C} 8$ & $0.6975(2)$ & 0.08981 (19) & $0.80433(8)$ & $0.0181(3)$ \\
\hline C9 & $0.5511(2)$ & $0.0862(2)$ & $0.74162(8)$ & $0.0204(3)$ \\
\hline C10 & $0.8194(2)$ & $0.24779(18)$ & $0.67912(8)$ & $0.0162(3)$ \\
\hline C11 & $1.2311(2)$ & $0.1686(2)$ & $0.96118(8)$ & 0.0218 \\
\hline C12 & $0.6629(3)$ & $0.3216(2)$ & $0.53901(9)$ & $0.0256(3)$ \\
\hline $\mathrm{H} 7$ & $0.658(3)$ & $0.041(2)$ & $0.8462(10)$ & $0.028(5)^{*}$ \\
\hline H8 & $0.400(3)$ & $0.022(2)$ & $0.7410(10)$ & $0.027(4) *$ \\
\hline H9 & $1.264(3)$ & $0.138(3)$ & $1.0104(11)$ & $0.035(5)^{*}$ \\
\hline $\mathrm{H} 10$ & $0.564(3)$ & $0.385(2)$ & $0.5600(10)$ & $0.035(5)^{*}$ \\
\hline H11 & $0.702(3)$ & $0.373(3)$ & $0.4949(11)$ & $0.037(5)^{*}$ \\
\hline H12 & $0.600(3)$ & $0.192(3)$ & $0.5294(10)$ & $0.038(5)^{*}$ \\
\hline
\end{tabular}

Atomic displacement parameters $\left(\AA^{2}\right)$

\begin{tabular}{lllllll}
\hline & $U^{11}$ & $U^{22}$ & $U^{33}$ & $U^{12}$ & $U^{13}$ & $U^{23}$ \\
\hline S1 & $0.02103(18)$ & $0.02210(18)$ & $0.01490(18)$ & $0.00253(13)$ & $-0.00004(13)$ & $0.00614(13)$ \\
N1 & $0.0152(5)$ & $0.0167(5)$ & $0.0130(5)$ & $0.0007(4)$ & $0.0036(4)$ & $0.0040(4)$ \\
N2 & $0.0216(6)$ & $0.0250(6)$ & $0.0152(6)$ & $0.0020(5)$ & $0.0040(5)$ & $0.0078(5)$
\end{tabular}




\begin{tabular}{|c|c|c|c|c|c|c|}
\hline N3 & $0.0155(6)$ & $0.0260(6)$ & $0.0190(6)$ & $-0.0011(5)$ & $0.0024(5)$ & $0.0049(5)$ \\
\hline N4 & $0.0203(6)$ & $0.0295(7)$ & $0.0186(6)$ & $0.0031(5)$ & $0.0005(5)$ & $0.0046(5)$ \\
\hline N5 & $0.0166(5)$ & $0.0159(5)$ & $0.0149(6)$ & $0.0028(4)$ & $0.0030(4)$ & $0.0019(4)$ \\
\hline N6 & $0.0182(6)$ & $0.0193(6)$ & $0.0212(6)$ & 0.0019 (4) & $0.0009(5)$ & $0.0034(5)$ \\
\hline $\mathrm{C} 1$ & $0.0155(6)$ & $0.0138(6)$ & $0.0150(6)$ & $0.0030(5)$ & $0.0025(5)$ & $0.0011(5)$ \\
\hline $\mathrm{C} 2$ & $0.0186(7)$ & $0.0180(7)$ & $0.0181(7)$ & $0.0012(5)$ & $0.0057(5)$ & $0.0042(5)$ \\
\hline $\mathrm{C} 3$ & $0.0168(7)$ & $0.0200(7)$ & $0.0232(8)$ & $0.0008(5)$ & $0.0039(6)$ & $0.0026(5)$ \\
\hline $\mathrm{C} 4$ & $0.0181(6)$ & $0.0149(6)$ & $0.0157(7)$ & $0.0034(5)$ & $0.0026(5)$ & $0.0020(5)$ \\
\hline C5 & $0.0221(7)$ & $0.0251(7)$ & $0.0153(7)$ & $0.0044(6)$ & $0.0022(6)$ & $0.0045(5)$ \\
\hline C6 & $0.0203(7)$ & $0.0305(8)$ & $0.0190(7)$ & $0.0053(6)$ & $0.0042(6)$ & $0.0093(6)$ \\
\hline S2 & 0.02219 (18) & $0.02074(18)$ & $0.01648(18)$ & $0.00230(13)$ & $0.00321(14)$ & $0.00600(13)$ \\
\hline N7 & $0.0134(5)$ & $0.0181(5)$ & $0.0149(6)$ & 0.0015 (4) & 0.0037 (4) & $0.0045(4)$ \\
\hline N8 & $0.0203(6)$ & $0.0281(7)$ & $0.0170(6)$ & $0.0028(5)$ & $0.0044(5)$ & $0.0090(5)$ \\
\hline N9 & $0.0152(6)$ & $0.0265(6)$ & $0.0213(6)$ & $-0.0005(5)$ & $0.0014(5)$ & $0.0057(5)$ \\
\hline N10 & $0.0182(6)$ & $0.0316(7)$ & $0.0203(7)$ & $0.0015(5)$ & $-0.0004(5)$ & $0.0048(5)$ \\
\hline N11 & 0.0159 (5) & $0.0160(5)$ & $0.0155(6)$ & $0.0021(4)$ & $0.0029(4)$ & $0.0033(4)$ \\
\hline N12 & $0.0170(6)$ & $0.0214(6)$ & $0.0184(6)$ & $0.0020(5)$ & $0.0026(5)$ & $0.0016(5)$ \\
\hline $\mathrm{C} 7$ & 0.0139 (6) & $0.0142(6)$ & $0.0160(6)$ & $0.0039(5)$ & $0.0019(5)$ & $0.0008(5)$ \\
\hline $\mathrm{C} 8$ & $0.0171(7)$ & $0.0200(7)$ & $0.0170(7)$ & $0.0018(5)$ & $0.0056(5)$ & $0.0036(5)$ \\
\hline $\mathrm{C} 9$ & $0.0164(7)$ & $0.0233(7)$ & $0.0206(7)$ & $0.0019(5)$ & $0.0034(6)$ & $0.0014(5)$ \\
\hline $\mathrm{C} 10$ & 0.0189 (7) & $0.0139(6)$ & $0.0160(7)$ & $0.0039(5)$ & $0.0023(5)$ & $0.0008(5)$ \\
\hline C11 & 0.0188 (7) & $0.0294(8)$ & $0.0168(7)$ & $0.0039(6)$ & $0.0023(6)$ & $0.0060(6)$ \\
\hline $\mathrm{C} 12$ & $0.0305(8)$ & $0.0281(8)$ & $0.0181(8)$ & $0.0075(7)$ & $-0.0006(6)$ & $0.0034(6)$ \\
\hline
\end{tabular}

Geometric parameters $\left(A,{ }^{o}\right)$

\begin{tabular}{llll}
\hline $\mathrm{S} 1-\mathrm{C} 4$ & $1.7453(15)$ & $\mathrm{S} 2-\mathrm{C} 10$ & $1.7487(15)$ \\
$\mathrm{S} 1-\mathrm{C} 6$ & $1.8004(16)$ & $\mathrm{S} 2-\mathrm{C} 12$ & $1.7992(16)$ \\
$\mathrm{N} 1-\mathrm{N} 3$ & $1.3311(16)$ & $\mathrm{N} 7-\mathrm{N} 9$ & $1.3314(16)$ \\
$\mathrm{N} 1-\mathrm{N} 2$ & $1.3412(16)$ & $\mathrm{N} 7-\mathrm{N} 8$ & $1.3421(16)$ \\
$\mathrm{N} 1-\mathrm{C} 1$ & $1.4347(17)$ & $\mathrm{N} 7-\mathrm{C} 7$ & $1.4291(17)$ \\
$\mathrm{N} 2-\mathrm{C} 5$ & $1.3207(19)$ & $\mathrm{N} 8-\mathrm{C} 11$ & $1.3182(19)$ \\
$\mathrm{N} 3-\mathrm{N} 4$ & $1.3176(17)$ & $\mathrm{N} 9-\mathrm{N} 10$ & $1.3148(17)$ \\
$\mathrm{N} 4-\mathrm{C} 5$ & $1.356(2)$ & $\mathrm{N} 10-\mathrm{C} 11$ & $1.3566(19)$ \\
$\mathrm{N} 5-\mathrm{C} 1$ & $1.3267(17)$ & $\mathrm{N} 11-\mathrm{C} 7$ & $1.3237(17)$ \\
$\mathrm{N} 5-\mathrm{C} 4$ & $1.3412(17)$ & $\mathrm{N} 11-\mathrm{C} 10$ & $1.3496(17)$ \\
$\mathrm{N} 6-\mathrm{C} 3$ & $1.3336(19)$ & $\mathrm{N} 12-\mathrm{C} 10$ & $1.3377(18)$ \\
$\mathrm{N} 6-\mathrm{C} 4$ & $1.3506(18)$ & $\mathrm{N} 12-\mathrm{C} 9$ & $1.3393(19)$ \\
$\mathrm{C} 1-\mathrm{C} 2$ & $1.3815(19)$ & $\mathrm{C} 7-\mathrm{C} 8$ & $1.3837(19)$ \\
$\mathrm{C} 2-\mathrm{C} 3$ & $1.393(2)$ & $\mathrm{C} 8-\mathrm{C} 9$ & $1.386(2)$ \\
$\mathrm{C} 2-\mathrm{H} 1$ & $0.885(19)$ & $\mathrm{C} 8-\mathrm{H} 7$ & $0.886(19)$ \\
$\mathrm{C} 3-\mathrm{H} 2$ & $0.951(17)$ & $\mathrm{C} 9-\mathrm{H} 8$ & $0.965(18)$ \\
$\mathrm{C} 5-\mathrm{H} 3$ & $0.891(19)$ & $\mathrm{C} 11-\mathrm{H} 9$ & $0.940(19)$ \\
$\mathrm{C} 6-\mathrm{H} 4$ & $0.974(17)$ & $\mathrm{C} 12-\mathrm{H} 10$ & $0.955(19)$ \\
$\mathrm{C} 6-\mathrm{H} 5$ & $0.988(17)$ & $\mathrm{C} 12-\mathrm{H} 11$ & $0.93(2)$ \\
$\mathrm{C} 6-\mathrm{H} 6$ & $0.96(2)$ & $\mathrm{C} 12-\mathrm{H} 12$ & $0.957(19)$ \\
& & & $101.37(8)$ \\
$\mathrm{C} 4-\mathrm{S} 1-\mathrm{C} 6$ & $101.92(7)$ & $\mathrm{C} 10-\mathrm{S} 2-\mathrm{C} 12$
\end{tabular}




\begin{tabular}{|c|c|c|c|}
\hline $\mathrm{N} 3-\mathrm{N} 1-\mathrm{N} 2$ & $113.78(11)$ & N9-N7-N8 & $113.63(11)$ \\
\hline $\mathrm{N} 3-\mathrm{N} 1-\mathrm{C} 1$ & $123.45(11)$ & N9-N7-C7 & $123.40(11)$ \\
\hline $\mathrm{N} 2-\mathrm{N} 1-\mathrm{C} 1$ & $122.75(11)$ & $\mathrm{N} 8-\mathrm{N} 7-\mathrm{C} 7$ & $122.96(11)$ \\
\hline $\mathrm{C} 5-\mathrm{N} 2-\mathrm{N} 1$ & $101.28(11)$ & $\mathrm{C} 11-\mathrm{N} 8-\mathrm{N} 7$ & $101.34(12)$ \\
\hline $\mathrm{N} 4-\mathrm{N} 3-\mathrm{N} 1$ & $105.82(12)$ & $\mathrm{N} 10-\mathrm{N} 9-\mathrm{N} 7$ & $105.89(12)$ \\
\hline $\mathrm{N} 3-\mathrm{N} 4-\mathrm{C} 5$ & 106.17 (12) & $\mathrm{N} 9-\mathrm{N} 10-\mathrm{C} 11$ & 106.19 (12) \\
\hline $\mathrm{C} 1-\mathrm{N} 5-\mathrm{C} 4$ & $114.47(12)$ & $\mathrm{C} 7-\mathrm{N} 11-\mathrm{C} 10$ & $114.67(12)$ \\
\hline $\mathrm{C} 3-\mathrm{N} 6-\mathrm{C} 4$ & $115.71(12)$ & $\mathrm{C} 10-\mathrm{N} 12-\mathrm{C} 9$ & $115.82(12)$ \\
\hline $\mathrm{N} 5-\mathrm{C} 1-\mathrm{C} 2$ & $125.31(12)$ & $\mathrm{N} 11-\mathrm{C} 7-\mathrm{C} 8$ & $125.20(12)$ \\
\hline $\mathrm{N} 5-\mathrm{C} 1-\mathrm{N} 1$ & $115.33(12)$ & $\mathrm{N} 11-\mathrm{C} 7-\mathrm{N} 7$ & $115.25(12)$ \\
\hline $\mathrm{C} 2-\mathrm{C} 1-\mathrm{N} 1$ & $119.36(12)$ & $\mathrm{C} 8-\mathrm{C} 7-\mathrm{N} 7$ & $119.55(12)$ \\
\hline $\mathrm{C} 1-\mathrm{C} 2-\mathrm{C} 3$ & $114.59(13)$ & $\mathrm{C} 7-\mathrm{C} 8-\mathrm{C} 9$ & $114.40(13)$ \\
\hline $\mathrm{C} 1-\mathrm{C} 2-\mathrm{H} 1$ & $122.4(11)$ & $\mathrm{C} 7-\mathrm{C} 8-\mathrm{H} 7$ & $122.5(11)$ \\
\hline $\mathrm{C} 3-\mathrm{C} 2-\mathrm{H} 1$ & $123.0(12)$ & $\mathrm{C} 9-\mathrm{C} 8-\mathrm{H} 7$ & $123.1(12)$ \\
\hline $\mathrm{N} 6-\mathrm{C} 3-\mathrm{C} 2$ & $123.19(13)$ & $\mathrm{N} 12-\mathrm{C} 9-\mathrm{C} 8$ & $123.46(14)$ \\
\hline $\mathrm{N} 6-\mathrm{C} 3-\mathrm{H} 2$ & $116.5(10)$ & $\mathrm{N} 12-\mathrm{C} 9-\mathrm{H} 8$ & $116.1(11)$ \\
\hline $\mathrm{C} 2-\mathrm{C} 3-\mathrm{H} 2$ & $120.3(10)$ & $\mathrm{C} 8-\mathrm{C} 9-\mathrm{H} 8$ & $120.5(11)$ \\
\hline $\mathrm{N} 5-\mathrm{C} 4-\mathrm{N} 6$ & $126.72(13)$ & $\mathrm{N} 12-\mathrm{C} 10-\mathrm{N} 11$ & $126.44(13)$ \\
\hline $\mathrm{N} 5-\mathrm{C} 4-\mathrm{S} 1$ & $119.87(10)$ & $\mathrm{N} 12-\mathrm{C} 10-\mathrm{S} 2$ & $119.96(10)$ \\
\hline $\mathrm{N} 6-\mathrm{C} 4-\mathrm{S} 1$ & $113.41(10)$ & $\mathrm{N} 11-\mathrm{C} 10-\mathrm{S} 2$ & $113.60(10)$ \\
\hline $\mathrm{N} 2-\mathrm{C} 5-\mathrm{N} 4$ & $112.96(13)$ & $\mathrm{N} 8-\mathrm{C} 11-\mathrm{N} 10$ & 112.94 (13) \\
\hline $\mathrm{N} 2-\mathrm{C} 5-\mathrm{H} 3$ & $124.0(12)$ & $\mathrm{N} 8-\mathrm{C} 11-\mathrm{H} 9$ & $124.6(12)$ \\
\hline $\mathrm{N} 4-\mathrm{C} 5-\mathrm{H} 3$ & $123.0(12)$ & $\mathrm{N} 10-\mathrm{C} 11-\mathrm{H} 9$ & $122.5(12)$ \\
\hline $\mathrm{S} 1-\mathrm{C} 6-\mathrm{H} 4$ & $108.9(11)$ & $\mathrm{S} 2-\mathrm{C} 12-\mathrm{H} 10$ & 109.7 (11) \\
\hline $\mathrm{S} 1-\mathrm{C} 6-\mathrm{H} 5$ & $110.3(10)$ & $\mathrm{S} 2-\mathrm{C} 12-\mathrm{H} 11$ & 105.7 (12) \\
\hline $\mathrm{H} 4-\mathrm{C} 6-\mathrm{H} 5$ & $112.5(14)$ & $\mathrm{H} 10-\mathrm{C} 12-\mathrm{H} 11$ & $110.6(16)$ \\
\hline $\mathrm{S} 1-\mathrm{C} 6-\mathrm{H} 6$ & $103.5(11)$ & $\mathrm{S} 2-\mathrm{C} 12-\mathrm{H} 12$ & $110.3(11)$ \\
\hline $\mathrm{H} 4-\mathrm{C} 6-\mathrm{H} 6$ & $110.8(15)$ & $\mathrm{H} 10-\mathrm{C} 12-\mathrm{H} 12$ & 111.9 (16) \\
\hline $\mathrm{H} 5-\mathrm{C} 6-\mathrm{H} 6$ & $110.4(15)$ & $\mathrm{H} 11-\mathrm{C} 12-\mathrm{H} 12$ & $108.4(16)$ \\
\hline
\end{tabular}

Hydrogen-bond geometry $\left(\AA,{ }^{\circ}\right)$

\begin{tabular}{lllll}
\hline$D-\mathrm{H} \cdots A$ & $D-\mathrm{H}$ & $\mathrm{H} \cdots A$ & $D \cdots A$ & $D-\mathrm{H} \cdots A$ \\
\hline $\mathrm{C} 2-\mathrm{H} 1 \cdots \mathrm{N} 9^{\mathrm{i}}$ & $0.89(2)$ & $2.58(2)$ & $3.203(2)$ & $129(2)$ \\
\hline
\end{tabular}

Symmetry code: (i) $x-1, y, z$. 